\title{
Material and Device Designs in Biomimetic Polymer Electronics
}

\author{
Sihong Wang \\ The University of Chicago \\ Eckhardt Research Center, Room 385 \\ $5640 \mathrm{~S}$ Ellis Ave. \\ Chicago, IL 60637 \\ sihongwang@uchicago.edu
}

\begin{abstract}
The vast amount of biological mysteries and biomedical challenges faced by human provide a prominent drive for seamlessly merging electronics with biological living systems (e.g. human bodies) to achieve long-term stable functions. Towards this trend, one of the key requirements for electronics is to possess biomimetic form-factors in various aspects for achieving the long-term biocompatibility. To enable such paradigm-shifting requirements, polymer-based electronics are uniquely promising for combining advanced electronic functionalities with biomimetic properties. In this talk, I will introduce our new molecular-design, chemical-synthesis and physical-processing concepts for polymer semiconductors, which enabled the incorporation of multiple biomimetic properties with advanced electronic functionalities. Fundamental understandings that have been obtained about the structure property relationship on these unprecedented material designs are serving as the foundation for opening up this new research direction for polymer semiconductors. Furthermore, enabled by these new materials, we have also created new device designs and fabrication processes for building unprecedented functional devices (i.e., sensors and circuits) that are intrinsically stretchable with unperturbed performance when operating conformably on human bodies. Collectively, our research is opening up a new generation of electronics that fundamentally change the way that the humans interact with electronics.
\end{abstract}

